\author{
Nota Científica \\ (Short Communication)
}

\title{
CONFIRMACIÓN DE LA PRESENCIA DE LA SERPIENTE DE CASCABEL CROTALUS SCUTULATUS PARA EL ESTADO DE MÉXICO, MÉXICO
}

\author{
Recibido: 10/06/2015; aceptado: 04/03/2016
}

Fernández-Badillo, L., Morales-Capellán, N., Contreras-Patiño, D. R. \& Carreño-Cervantes, A. 2016. Confirmación de la presencia de la serpiente de cascabel Crotalus scutulatus para el Estado de México, México. [Confirmation of the presence of rattlesnake Crotalus scutulatus for the State of Mexico, Mexico]. Acta Zoológica Mexicana (n.s.), 32(2): 202-205.

\begin{abstract}
We present a new record of rattlesnake Crotalus scutulatus collected in the county of La Paz, Mexico, that confirms the presence of the species for the Mexican state of Mexico. There is a dubious prior record of a specimen collected on 1967. Either way this species has not been collected again until now.
\end{abstract}

Crotalus scutulatus (Kennicott, 1861) se distribuye desde el oeste del desierto de Mojave en Estados Unidos de Norte América hasta el Valle de Tehuacán, al sur de Puebla, México (Campbell \& Lamar 2004; Canseco-Márquez \& Gutiérrez-Mayen 2010). Sin embargo, los registros para la parte centro-sur del país son pocos, específicamente en los estados de Guanajuato, Querétaro, Hidalgo, Tlaxcala y Veracruz (Pérez-Higareda \& Smith 1991; Fernández et al. 2006; Domínguez-Laso et al. 2007; Dixon \& LemosEspinal 2010; Fernández-Badillo et al. 2011). Para el Estado de México, Campbell \& Lamar (2004) mencionan la probable presencia de la especie al noreste del estado. En ese mismo año Matías-Ferrer \& Murillo (2004) registraron un ejemplar recolectado en 1967 en la localidad de Cerro del Pino, Ixtapaluca, al este del Estado de México a 2500 m (IPN ENCB 6276), con las coordenadas $19^{\circ} 20^{\prime} \mathrm{N},-99^{\circ} 55^{\prime} 18^{\prime \prime} \mathrm{W}$, sin embargo, éstas difieren de las coordenadas correctas para la localidad El Pino (19 $20^{\prime} \mathrm{N},-98^{\circ} 55^{\prime} 18^{\prime \prime} \mathrm{W}$ ) y corresponden más bien a un sitio al oeste del Estado de México, a $28.68 \mathrm{~km}$ al noroeste de la Ciudad de Toluca y a una altitud de $2851 \mathrm{~m}$. Ambas localidades difieren únicamente en un grado en la longitud, por lo que seguramente la imprecisión se debe a un error de transcripción en la coordenada.

Es importante mencionar, que en el listado más reciente para el estado de México no se considera la presencia de C. scutulatus (Aguilar-Miguel et al. 2009).
En el presente trabajo se registran dos hembras de $C$. scutulatus (Figs. 1a y 1b), procedentes de El Pino, Los Reyes Acaquilpan, municipio de La Paz, México, a 2377 m (19²1'12.65” N, -98 56'33.17” W; WGS84). Este sitio se ubica en el este-centro del Estado de México, a $21 \mathrm{~km}$ al sureste del centro de la Ciudad de México y se encuentra aproximadamente a $2.5 \mathrm{~km}$ de la localidad mencionada por Matías-Ferrer \& Murillo (2004). El clima en el municipio de La Paz, es templado subhúmedo con lluvias en verano (INEGI 2009), y de acuerdo con la estación meteorológica Los Reyes, ubicada $5.21 \mathrm{~km}$ al oeste de la localidad de recolecta, la temperatura media anual es de $16.5^{\circ} \mathrm{C}$ y la precipitación anual de $509 \mathrm{~mm}$ (SMN 2015). La vegetación en el sitio se conforma de pastizales y zonas con vegetación perturbada.

Los ejemplares se depositaron en el Predio Intensivo de Manejo de Vida Silvestre X-Plora Reptilia (XPR), en resguardo permanente mediante el acta de canalización CIVSLRC081014; Los ejemplares presentan una longitud hocico-cloaca (LHC) de $560 \mathrm{~mm}$ y longitud de la cola (LC) de 50 mm (XPR-049) y una LHC de $600 \mathrm{~mm}$ y una LC de $60 \mathrm{~mm}$ (XPR-050), respectivamente. En cuanto a las escamas, presentan 160 ventrales y 24 subcaudales (XPR-049) y 164 ventrales y 24 subcaudales (XPR-050). Ambos presentan 13 escamas supralabiales y 15 infralabiales a ambos lados de la cabeza y dos escamas intersupraoculares; las cantales posteriores son grandes y las porciones laterales se extienden ventralmente sobre el canto rostral y toman el lugar de una loreal superior, y junto con la loreal separan la preocular superior y la postnasal; presentan también una escama plana en forma de luna creciente que bordea posteromedialmente cada supraocular. Lo anterior concuerda con lo descrito por Campbell y Lamar (2004) para C. scutulatus. La identificación fue corroborada por el M. en C. Luis Canseco-Márquez.

Para verificar si los nuevos registros concuerdan con la distribución potencial de C. scutulatus, se elaboró un modelo de nicho ecológico con el algoritmo de MaxEnt, sin incluir dichos registros y utilizando registros de presencia 


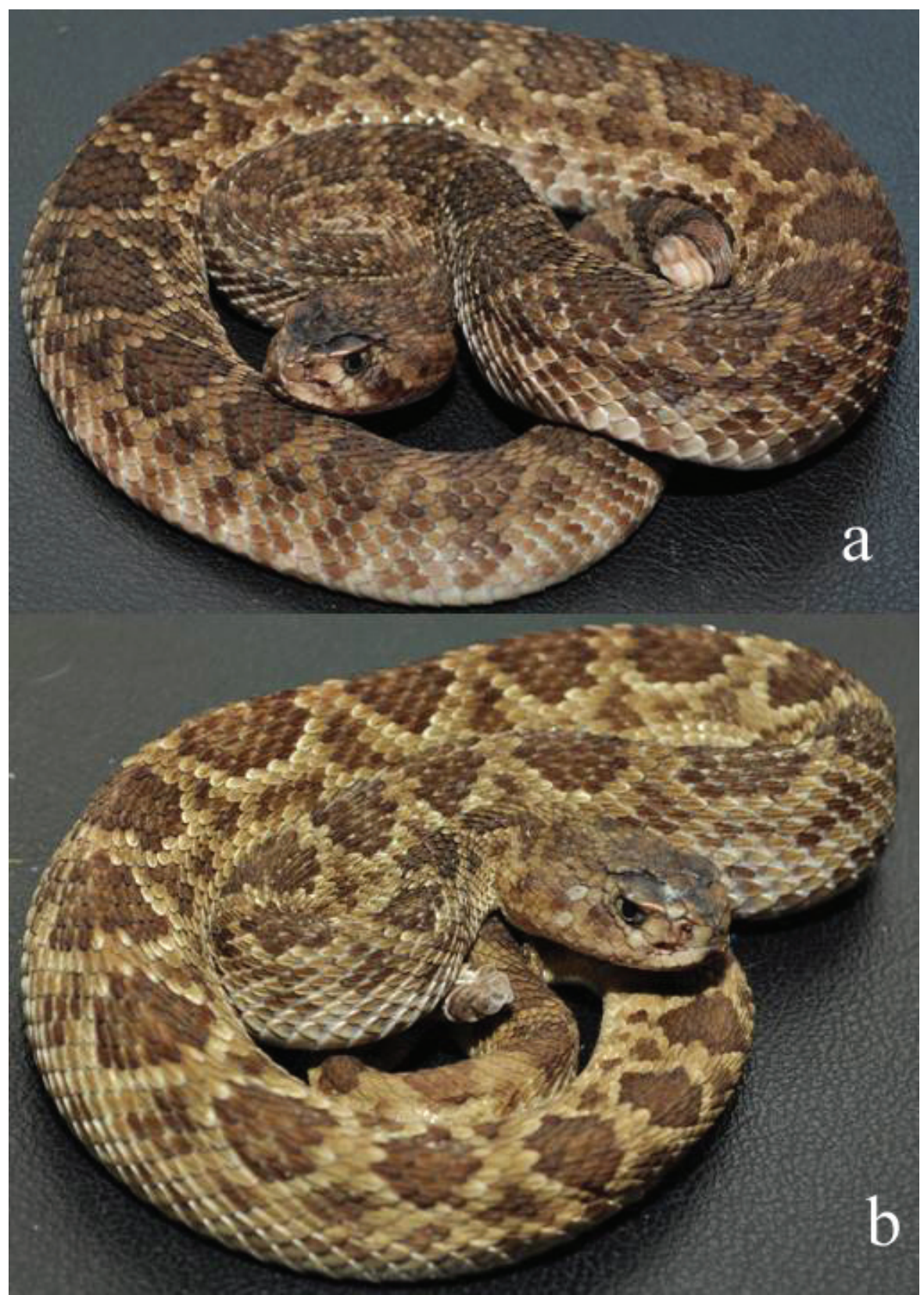

Figura 1. a) Crotalus scutulatus hembra (XPR-049); b) C. scutulatus hembra (XPR-050); ambas recolectadas en la localidad de El Pino, Los Reyes Acaquilpan, municipio de La Paz, México.

de la especie para todo México, obtenidos de literatura y colecciones en línea (REMIB, GBIF, Herpnet). Se utilizaron ocho variables climáticas (WorldClim) no correlacionadas, seleccionadas a partir de un análisis multifactorial. El área accesible para México se construyó con el bioma “zonas áridas” (Olson et al. 2001). El modelo resultante (Fig. 2), se transformó en un mapa binario (presencia/ausencia), con un umbral de corte de omisión (entrenamien- to y validación) menor al $10 \%$ al cual se le sobrepusieron los registros encontrados, así como el de Matías-Ferrer \& Murillo (2004) y el registro fósil mencionado por Brattstrom (1975) en el municipio de Tequixquiac, distrito de Zumpango.

El modelo obtenido predice la presencia al noreste del D.F. y en algunas zonas de las regiones de Amecameca, Nezahualcóyotl y Texcoco, Estado de México. Sin em- 


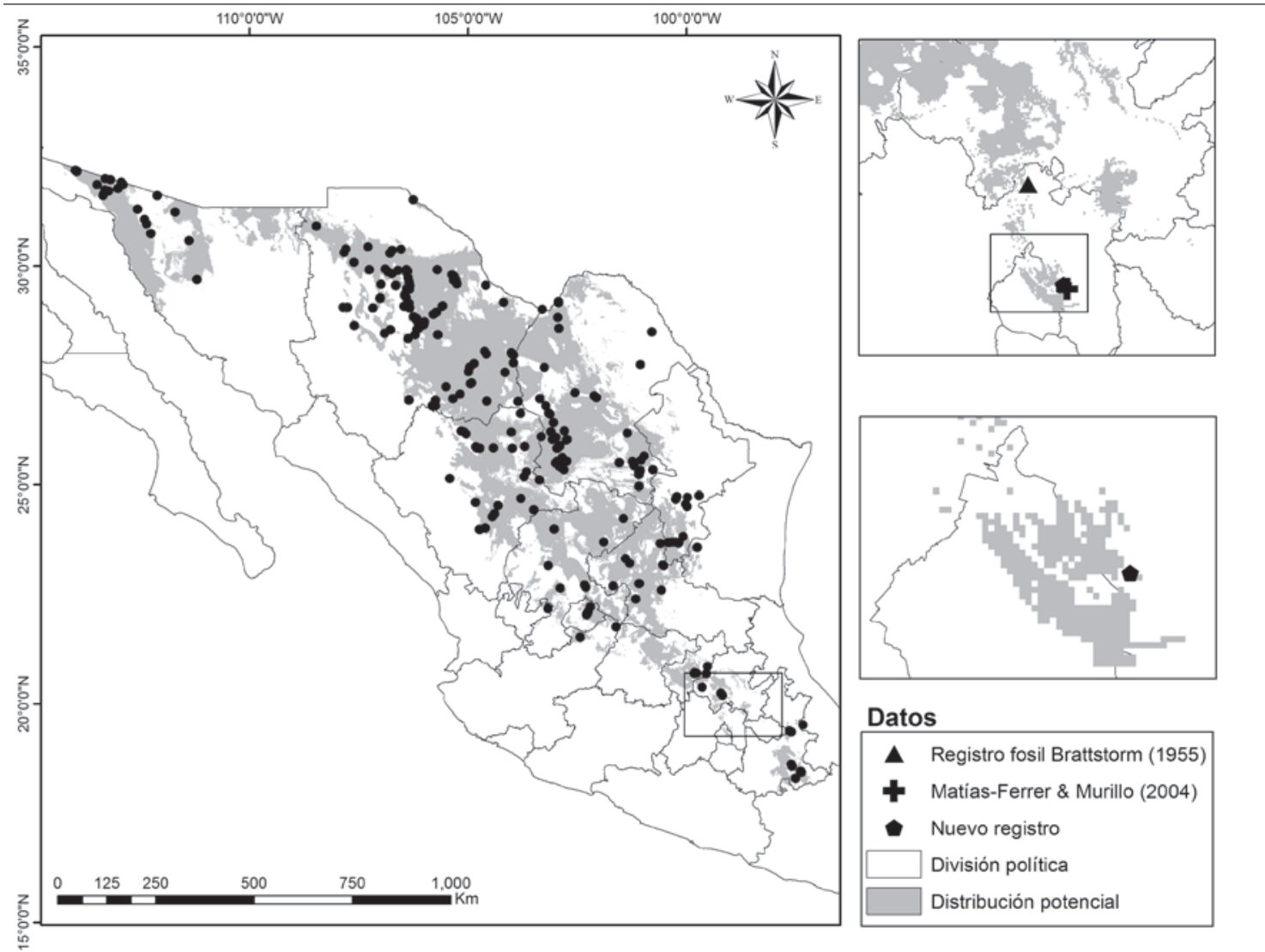

Figura 2. Mapa de distribución potencial de Crotalus scutulatus, así como los nuevos registros de la localidad El Pino, Los Reyes Acaquilpan, municipio de La Paz, México, y los registros previos para el Estado de México.

bargo, al analizar a detalle esta zona, se detectó que el nuevo registro se localiza aproximadamente a $1 \mathrm{~km}$ fuera de la predicción, esto puede deberse a que los modelos de nicho ecológico son una representación de la idoneidad ambiental (Mateo et al. 2011), lo cual no implica que por necesidades ecológicas (forrajeo, hibernación, búsqueda de pareja) los individuos no puedan moverse hacia zonas subóptimas (ambientalmente) ubicadas fuera de las áreas de predicción. En este sentido, se han registrado desplazamientos en individuos de C. atrox, de hasta 3.5 km durante las migraciones otoñales (Landreth 1973). Por lo tanto, es probable que los ejemplares encontrados de C. scutulatus pudieron haberse desplazado más de un kilómetro fuera del área de predicción.

Los registros encontrados son relevantes ya que desde 1967 no se registraba a C. scutulatus en el Estado de México. Además de la confirmación de la presencia de la especie para la entidad, se amplía su distribución hacia la zona este-centro del estado y no únicamente en el noreste como lo suponían Campbell \& Lamar (2004).
AGRADECIMIENTOS. Los autores agradecen a Cristián Raúl Olvera Olvera por el apoyo en el traslado de los ejemplares del CIVSLos Reyes, al PIMVS XPR. Al proyecto "Diversidad Biológica del Estado de Hidalgo (tercera etapa) FOMIX-CONACyT-HGO-2012191908” por el apoyo logístico. A Hublester Domínguez-Vega y Carlos Maciel-Mata por su apoyo en la elaboración del modelo y la revisión del manuscrito. A dos revisores anónimos por sus valiosos comentarios.

\section{LITERATURA CITADA}

Aguilar-Miguel, X., Casas-Andreu, G., Cárdenas-Ramos, P. J \& Cantellano de Rosas, E. 2009. Análisis espacial y conservación de los anfibios y reptiles del estado de México. Ciencia Ergo Sum, 16: 171-180.

Brattstrom, B. H. 1955. Records of some Pliocene and Pleistocene reptiles and amphibians from Mexico. Bulletin of Southern California Academy of Sciences, 54: 1-4.

Campbell. J. A. \& Lamar, W. W. 2004. The venomous reptiles of the western hemisphere. Cornell University Press, Ithaca, New York, U.S.A., 870 pp. 
Canseco-Márquez, L. \& Gutiérrez-Mayén, M. G. 2010. Anfibios y reptiles del Valle de Tehuacán-Cuicatlán. CONABIO-Cuicatlán A.C.-BUAP, México, 302 pp.

Dixon, J. R. \& Lemos-Espinal, J. A. 2010. Anfibios y reptiles del estado de Querétaro, México. Texas A \& M University, UNAMCONABIO, México, 428 pp.

Domínguez-Laso, M., García-Padilla, U., Pérez-Ramos, E. \& Quijada-Mascareñas, A. 2007. Geographic distribution: Serpentes: Crotalus scutulatus salvini. Herpetological Review, 38: 485.

Fernández, A. J., Sánchez, O. \& Flores-Villela, O. 2006. Anfibios y reptiles del estado de Tlaxcala, pp. 224-240. In: A. Ramírez-Bautista, L. Canseco-Márquez \& F. Mendoza-Quijano (Eds.). Inventarios herpetofaunísticos de México: Avances en el conocimiento de su biodiversidad. Publicaciones de la Sociedad Herpetológica Mexicana, México.

Fernández-Badillo, L., Morales-Capellán, N. \& Goyenechea, I. 2011. Serpientes venenosas del estado de Hidalgo. UAEH, Hidalgo, México, 98 pp.

INEGI. 2009. Prontuario de información geográfica municipal de los Estados Unidos Mexicanos. La Paz, México. Clave geoestadística 15070. INEGI, México.

Landreth, H. M. 1973. Orientation and behavior of the Rattlesnake, Crotalus atrox. Copeia, 1973: 26-31.

Mateo, R. G., Felicísimo, A. M. \& Muñoz, J. 2011. Modelos de distribución de especies: Una revisión sintética. Revista Chilena de Historia Natural, 84: 217-240.

Matías-Ferrer, N. \& Murillo, S. 2004. Geographic distribution: Serpentes Crotalus scutulatus. Herpetological Review, 35: 191.

Olson, D. M., Dinerstein, E., Wikramanayake, E. D., Burgess, N.
D., Powell, G. V. N., Underwood, E. C., D’Amico, J. A., Itoua, I., Strand, H. E., Morrison, J. C., Loucks, C. J., Allnut, T. F., Ricketts, T. H., Kura, Y., Lamoreux, J. F., Wettengel, W. W., Hedao, P. \& Kassem, K. R. 2001. Terrestrial ecoregions of the world: a new map of life on Earth. Bioscience, 51: 933-938.

Pérez-Higareda, G., \& Smith, H. M. 1991. Ofidiofauna de Veracruz. Análisis taxonómico y zoogeográfico. Publicaciones especiales del Instituto de Biología 7. UNAM, México, 122 pp.

SMN. 2007. Servicio Meteorológico Nacional. Normales climatológicas, estado de México. Disponible en: http://smn.cna.gob.mx (revisado en febrero de 2016).

\section{LEONARDO FERNÁNDEZ-BADILLO, ${ }^{1,2, *}$ NALLELY MORALES-CAPELLÁN ${ }^{2}$ DANIEL RAFAEL CONTRERAS-PATIÑO ${ }^{3}$ y ANGÉLICA CARREÑO-CERVANTES ${ }^{3}$}

${ }^{1}$ Centro de Investigaciones Biológicas, Universidad Autónoma del Estado de Hidalgo, Ciudad del Conocimiento. Km 4.5 Carr. PachucaTulancingo, s/n, Mineral de la Reforma, Hidalgo. C. P. 42184.

${ }^{2}$ Predio Intensivo de Manejo de Vida Silvestre X-Plora Reptilia, Carretera México-Tampico s/n, Pilas y Granadas, Metztitlán, Hidalgo, México. C. P. 43350 <xplorareptilia@hotmail.com> ${ }^{3}$ Centro para la Conservación e Investigación de la Vida Silvestre, SEMARNAT. Circuito Emiliano Zapata Norte, El Pino, Los Reyes Acaquilpan, Estado de México. C.P 56507.

*correspondencia <cyrtopsis@hotmail.com> 